\title{
EAl Endorsed Transactions

\section{An approach to reduce data dimension in building effective Network Intrusion Detection Systems}

\author{
Hoang Ngoc Thanh ${ }^{1, *}$, Tran Van Lang ${ }^{2}$ \\ ${ }^{1}$ Lac Hong University, Vietnam \\ ${ }^{2}$ Institute of Applied Mechanics and Informatics, VAST, Vietnam
}

\section{Abstract}

The main function of the network Intrusion Detection System (IDS) is to protect the system, analyze and predict network access behavior of users. These behaviors are considered normal or an attack. Machine learning methods (ML) are used in IDSs because of the ability to learn from past attack patterns to recognize new attack patterns. These methods are effective but have relatively high computational costs. Meanwhile, the traffic of network data is growing rapidly, the computational cost issues need to be addressed. This paper addresses the use of algorithms combined with information metrics to reduce the features of the dataset to be analyzed. As the result, it helps to build IDSs with lower cost but higher performance suitable for large scale networks. The test results on the UNSW-NB15 dataset demonstrate: with the optimal set of features suitable for the attack type as well as the machine learning method, the quality of classification is improved with less training and testing time.

Received on 27 July 2019; accepted on 05 August 2019; published on 20 August 2019

Keywords: Intrusion Detection System, Machine learning, Feature selection, UNSW-NB15 dataset.

Copyright $\odot 2019$ Hoang Ngoc Thanh et al., licensed to EAI. This is an open access article distributed under the terms of the Creative Commons Attribution license (http://creativecommons.org/licenses/by/3.0/), which permits unlimited use, distribution and reproduction in any medium so long as the original work is properly cited.

doi:10.4108/eai.13-7-2018.162633

\section{Introduction}

Due to recent technological advances, network-based services increasingly play an important role in modern society. Intruders are constantly looking for vulnerabilities on the computer system to gain unauthorized access to the kernel of the system. However, existing IDSs are still not flexible enough, scalability is not high, nor is it strong enough to deal with such attacks.

Previously, law-based methods were dominant. These methods find intrusion by comparing the characteristics of the data to be analyzed with known attack signs. As network traffic grows rapidly, updating the attack signs is becoming more and more difficult, tedious and time-consuming. Since then, machine learning methods have been introduced to solve intrusion detection problems. Machine learning refers to computer algorithms that are capable of learning from past attack patterns to recognize new attack patterns. Based on machine learning, IDSs have performed better in many reports as well as actual implementations [1]. However, the "no

${ }^{*}$ Corresponding author. Email: thanhhn@bvu.edu.vn model" assets of such methods causes relatively high computational costs. Moreover, the traffic of network data is growing rapidly, computer cost issues need to be resolved [2].

One of the important solutions to reduce computational costs is to select the best features of the data to be analyzed. Reducing the number of features of a dataset will reduce training and testing time. At the same time, it improves the performance of classifiers in IDS. There are multiple feature selection methods, broadly categorized into Filter, Wrapper and Embedded methods based on their interaction with the predictor during the selection process. The Filter methods rank the variables as a preprocessing step, and feature selection is done before choosing the model. In the Wrapper approach, nested subsets of variables are tested to select the optimal subset that work best for the model during the learning process. The Embedded methods are those which incorporate variable selection in the training algorithm.

The content of this paper proposes a new method to reduce the features of a dataset based on Information Gain (IG), Gain Ratio (GR) and Correlation Attribute 
(CA) using the Wrapper approach. The comparative results show that the proposed method gives better performance than the other existing methods.

The remainder of this paper is organized as follows, Section 2 presents related works, Section 3 presents the challenges posed by the problem and proposed solution, Section 4 presents proposed feature selection method for improving classification efficiency with the UNSW-NB15 dataset, Section 5 presents the results obtained through experiments and Section 6 is conclusions and issues that need to be researched in the future.

\section{Related works}

The use of data dimension reduction techniques to enhance the effectiveness of IDS, has many different approaches presented by scholars, can be categorized into Bio-Inspired and Non-inspired Algorithms.

\subsection{Bio-Inspired Algorithms}

In Aslahi et al. [3] research a hybrid technique of Support Vector Machine (SVM) and GA was proposed for IDS. The proposed mix algorithm was utilized in decreasing the quantity of features from 41 to 10. Features sorted into three groups using GA algorithm from the most important feature group to less important feature groups. This is done such that 4 features are set in the highest significance, 4 included in the next, and 2 included in the third significance. The outcomes show that the proposed hybrid algorithm can achieve a true positive estimation of 0.973 , while the false positive rate was 0.017 .

Alternatively, a network intrusion detection method combining ant colony algorithm to select the features with a feature weighting SVM proposed by Xingzhu et al. [4]. First, the use of SVM classification accuracy and feature subset dimension construct a comprehensive fitness weighting index. Then use the ant colony algorithm for global optimization and multiple search capabilities to achieve optimal solutions feature subset search feature. And then selected the key feature of network data and calculated information gain access to various features weights and heavy weights to build SVM classifier based on the characteristics of network attacks right. At last, refine the final design of the local search methods to make the feature selection results without redundant features while improve the convergence resistance, and verify the dataset by KDD99 effectiveness of the algorithm. The results exhibited that the proposed approach can successfully reduce the dimension of features and have enhanced network intrusion detection accuracy to $95.75 \%$

Rani et al. [5] proposed a new hybrid intrusion detection method combining multiple classifiers for classifying anomalous and normal activities on the computer network is presented. The misuse detection model is built based on the C5.0 Decision tree algorithm and using the information collected anomaly detection model is built which is implemented by one-class SVM. Integration of multiple algorithms helps to get better performance. The experimental results are performed on NSL-KDD dataset, and it is shown that overall performance of the proposed approach is improved in terms of detection rate and low false alarms rate in comparison to the existing techniques.

Ghanem et al. [6] anticipated a novel approach based on multi-objective artificial bee colony (ABC) for feature selection, particularly for intrusion-detection systems. The approach is divided into two stages: generating the feature subsets of the Pareto front of non-dominated solutions in the first stage and using the hybrid ABC and particle swarm optimization (PSO) with a feedforward neural network (FFNN) as a classifier to evaluate feature subsets in the second stage. Thus, the proposed approach consists of two stages: (1) using a new feature selection technique called multi-objective $\mathrm{ABC}$ feature selection to reduce the number of features of network traffic data and (2) using a new classification technique called hybrid ABC-PSO optimized FFNN to classify the output data from the previous stage, determine an intruder packet, and detect known and unknown intruders. The proposed approach did not only provide a new approach for feature selection but also proposed a new fitness function for feature selection to diminish the number of features and achieve the minimum rate of classification errors and false alarms.

Acharya et al. [7] proposed an intelligent water drops (IWD) algorithm - based feature selection method. This method uses the IWD algorithm, a nature-inspired optimization algorithm for the feature subset selection along with SVM as a classifier for evaluation of the features selected. The experiments are conducted using KDD99 dataset. From 41 to 9, the model substantially reduced the features. Parameters found to have been better improved as presented in the new model with a proposed method are precision, false alarm rate accuracy and rate of detection. This outcome measured improved than other prevailing models. A precision rate of $99.4075 \%$ and an accuracy score of $99.0915 \%$ were recorded on the new model. While a low false rate of $1.405 \%$ and a precision rate of $99.108 \%$ were also recorded.

\subsection{Non-inspired Algorithms}

Ganapathy et al. [8] developed a new intelligent Conditional Random Field (CRF) based feature selection algorithm to optimize the number of features. In addition, an existing Layered Approach (LA) based algorithm is used to perform classification with these reduced features. The KDD99 dataset benchmark was used in 
this research and the experimental results showed that the accuracy for different types of attack was (probe $=$ 99.98, $\mathrm{DOS}=97.62, \mathrm{R} 2 \mathrm{~L}=32.43, \mathrm{U} 2 \mathrm{R}=86.91)$.

Ambusaidi et al. [9] proposed a mutual information based algorithm that analytically selects the optimal feature for classification. This algorithm can handle linearly and nonlinearly dependent data features. Its effectiveness is evaluated in the cases of network intrusion detection. Least Square Support Vector Machine based IDS (LSSVM-IDS), is built using the features selected by their feature selection algorithm. The performance of LSSVM-IDS is evaluated using three datasets: KDD99, NSL-KDD and Kyoto 2006. The evaluation results show that their feature selection algorithm contributes more critical features for LSSVM-IDS to achieve better accuracy and lower computational cost compared with other methods.

Madbouly et al. [10] proposed an upgraded model to choose an arrangement of the most applicable features to increase attack detection precision and enhance general system performance. In their approach, the KDD99 dataset was utilized, and the chosen important features containing just 12 -beyond the 41 -full features set. This now decreases the magnitude of the KDD99 workbench dataset by over $70 \%$. They gauged the performance of the proposed model and confirmed its viability and attainability by comparing it with nine-unique models and with a model that utilized the 41 -features dataset. The experimental outcomes demonstrated that, their improved prototype could productively accomplish increased detection rate, performance rate, low false alarm rate, and quick and consistent detection process.

\section{Preliminary}

The above known researches still have problems that need to be solved:

(1) The dataset used in the experiments is KDD99 or NSL-KDD, these are datasets that are over 20 years old, so it does not contain modern normal activities and contemporary synthetic attack behaviors.

(2) The evaluation metrics used by the majority of authors is not suitable for the imbalanced datasets as in network intrusion detection problem.

(3) Known feature reduction algorithms have low accuracy and high false alarm rates.

\section{Proposed method}

This paper proposes to resolve the above issues by the following methods:

(1) Use the new UNSW-NB15 dataset [1] created by the Australian Network Security Center (ACSC) in 2015.

(2) Use evaluation metrics such as $F-$ Measure, GMeans especially suitable for imbalanced datasets.

(3) Use the IG, GR and CA combined with the Backward
Feature Elimination (BFE) algorithm to effectively reduce the features.

To find the set of features best suited to the type of attack as well as the machine learning method. First, depending on the type of attack, features will be ordered (descending) based on IG, GR and CA. Then, BFE algorithm is applied to select the most appropriate features for each machine learning method. The following section gives a brief overview of measures used for ranking features, machine learning models, evaluation metrics, as well as the algorithms to select features used in the experiments.

\subsection{Measures used for ranking features}

The proposed measures to rank features include: Information Gain, Gain Ratio and Correlation Attribute are defined as follows.

Let $S$ be set consisting of $s$ data instances with $m$ distinct classes. The expected information needed to classify a given instance is given by

$$
I(S)=-\sum_{i=1}^{m} p_{i} \log _{2} p_{i}
$$

where $p_{i}$ is the probability that an arbitrary instance belongs to class $C_{i}$ and is estimated by $s_{i} / s$.

Let attribute $A$ has $v$ distinct values. Let $s_{i j}$ be number of instances of class $C_{i}$ in a subset $S_{j} . S_{j}$ contains those instances in $S$ that have value $a_{j}$ of $A$. The entropy, or expected information based on the partitioning into subsets by $A$, is given by

$$
\operatorname{Ent}(A)=-\sum_{i=1}^{m} I(S) \frac{s_{1 i}+s_{2 i}+\ldots+s_{m i}}{s}
$$

(1) The Information Gain on feature $A$ is calculated as follows [11]:

$$
\operatorname{Gain}(A)=I(S)-\operatorname{Ent}(A)
$$

The value represents the information generated by splitting the training dataset $S$ into $v$ partitions corresponding to $v$ outcomes of a test on the attribute A:

$$
\text { SplitInfo }(A)=-\sum_{i=1}^{v} \frac{\left|S_{i}\right|}{|S|} \log _{2} \frac{\left|S_{i}\right|}{|S|}
$$

(2) The Gain Ratio is defined as [11]:

$$
\operatorname{GainRatio}(A)=\operatorname{Gain}(A) / \operatorname{SplitInfo}(A)
$$

(3) Attribute Correlation specifies the degree of dependency between attributes, it represents a linear relationship between attributes [11]:

$$
r_{a b}=\frac{\sum_{i=1}^{n}\left(a_{i}-\bar{A}\right)\left(b_{i}-\bar{B}\right)}{N \sigma_{A} \sigma_{B}}
$$


Here, $N$ is the number of instances, $a_{i}$ and $b_{i}$ are the corresponding values of $A$ and $B$ in the $i_{t h}$ instance, $\bar{A}$ and $\bar{B}$ are the mean values of $A$ and $B ; \sigma_{A}$ and $\sigma_{B}$ are the standard deviations of $A$ and $B$.

\subsection{Machine learning models}

In the experiments of this paper, the machine learning models used include: K Nearest Neighbors (kNN), Support Vector Machine (SVM), Artificial Neural Network (ANN), Decision Tree (DT), Naive Bayes (NB) and Logistic Regression (LR).

\subsection{Use the right evaluation metrics}

Using Accuracy to assess the quality of classifiers has been used by many scholars. However, with imbalanced data, using Accuracy to evaluate the quality of the classifiers is not really effective. Therefore, more comprehensive metrics have been suggested for evaluation such as F-Measure, G-Means [12], [13].

\subsection{Algorithms using BFE combine with IG, GR and $\mathrm{CA}$ to reduce features}

The BFE algorithm is implemented as follows: First, the features of the UNSW-NB15 dataset are calculated for IG, GR and CA. The features will then be sorted in descending order of IG, GR and CA. A classification is built on the training dataset and is evaluated on the testing dataset with all features. After that, the features will then be removed one by one starting with the least important feature (with the lowest values of IG, GR and $\mathrm{CA}$ ), then the classifier will be trained and tested on the training and testing datasets with the set of features is reduced, if the quality of the classification is improved, the feature will be removed, otherwise the feature will be retained. The pseudo code of the BFE algorithm is as follows:

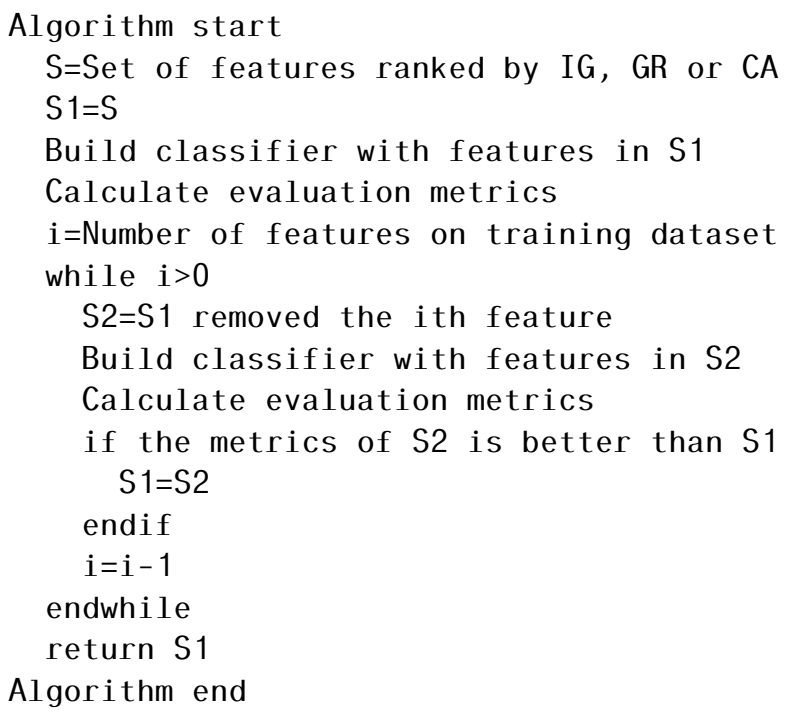

\section{EXPERIMENTS}

Programs and algorithms in the experiment using the Java programming language, based on the library, Weka machine learning framework developed by Waikato University, New Zealand.

Part 5.1 presents the datasets used in the experiment.

Part 5.2 presents the evaluation metrics used in the experiment.

Part 5.3 presents the results obtained using BFE combined with IG, GR and CA to reduce features with attack classes such as Worms, Shellcode, Backdoor, Generic, ...

\subsection{Datasets}

About the datasets used in IDS: KDD99, NSL-KDD and UNSW-NB15 are the most popular datasets, according to statistics from 2015 to 2018, showed that the NSL-KDD dataset is used $38 \%$, KDD99 is $23 \%$ and UNSW-NB15 is $12 \%$ [1]. Previously, machine learning and data dimensional reduction techniques with the KDD99, NSL-KDD datasets are also performed by the authors [14-16]. In this paper, the UNSW-NB15 dataset was used for experiment because of its outstanding advantages compared to the KDD99 and NSL-KDD datasets [17]. Furthermore, studies of IDS from 2015 to 2018 show the low classification efficiency of their methods with the UNSW-NB15 dataset [1]. So a problem is to improve the classification method so that the results are better on this dataset.

The UNSW-NB15 dataset was developed using the IXIA tool to extract modern and offensive behavior conducted by ACSC in 2015. This dataset includes 9 types of attacks, 49 features and 2,540,044 instances [17], Table 1 describes the order and names of the features. A part of this dataset is divided into training and testing datasets, which are used extensively in scholars' experiments, detailed information about the datasets is presented in Table 2, with 9 types of attacks in dataset include: Analysis, Backdoor, DoS, Exploits, Fuzzers, Generic, Reconnaissance, Shellcode and Worms. In the experiments, to avoid overfitting, we do not use kfold cross validation but use training dataset $(82.332$ instances) and test dataset (175.341 instances) separately included in UNSW-NB15.

The UNSW-NB15 dataset has several advantages when compared to the NSL-KDD dataset. First, it contains modern normal behavior and contemporary general attack activities. Second, the probability distribution of training and test datasets is similar. Third, it includes a set of features from payload and header of packages to reflect effective network packets. Finally, the complexity of evaluating UNSW-NB15 for existing classification systems shows that this dataset has complex patterns. This means that the dataset can be used to evaluate current and new classification methods 
Table 1. The features of UNSW-NB15 dataset.

\begin{tabular}{|c|l|c|l|l|l|}
\hline ID & Feature & ID & Feature & ID & Feature \\
\hline 1 & attack_cat & 16 & dloss & 31 & response_body_len \\
\hline 2 & dur & 17 & sinpkt & 32 & ct_srv_src \\
\hline 3 & proto & 18 & dinpkt & 33 & ct_state_ttl \\
\hline 4 & service & 19 & sjit & 34 & ct_dst_ltm \\
\hline 5 & state & 20 & djit & 35 & ct_src_dport_ltm \\
\hline 6 & spkts & 21 & swin & 36 & ct_dst_sport_ltm \\
\hline 7 & dpkts & 22 & stcpb & 37 & ct_dst_src_ltm \\
\hline 8 & sbytes & 23 & dtrcpb & 38 & is_ftp_login \\
\hline 9 & dbytes & 24 & dwin & 39 & ct_ftp_cmd \\
\hline 10 & rate & 25 & tcprtt & 40 & ct_flw_http_mthd \\
\hline 11 & sttl & 26 & synack & 41 & ct_src_ltm \\
\hline 12 & dttl & 27 & ackdat & 42 & ct_srv_dst \\
\hline 13 & sload & 28 & smean & 43 & is_sm_ips_ports \\
\hline 14 & dload & 29 & dmean & & \\
\hline 15 & sloss & 30 & trans_depth & & \\
\hline
\end{tabular}

Table 2. Information about UNSW-NB15 traning and testing datasets.

\begin{tabular}{|l|c|c|c|c|}
\hline Types of attacks & \multicolumn{2}{|c|}{ Testing dataset } & \multicolumn{2}{c|}{ Training dataset } \\
\hline Normal & 56.000 & $31,94 \%$ & 37.000 & $44,94 \%$ \\
\hline Analysis & 2.000 & $1,14 \%$ & 677 & $0,82 \%$ \\
\hline Backdoor & 1.746 & $1,00 \%$ & 583 & $0,71 \%$ \\
\hline DoS & 12.264 & $6,99 \%$ & 4.089 & $4,97 \%$ \\
\hline Exploits & 33.393 & $19,04 \%$ & 11.132 & $13,52 \%$ \\
\hline Fuzzers & 18.184 & $10,37 \%$ & 6.062 & $7,36 \%$ \\
\hline Generic & 40.000 & $22,81 \%$ & 18.871 & $22,92 \%$ \\
\hline Reconnaissance & 10.491 & $5,98 \%$ & 3.496 & $4,25 \%$ \\
\hline Shellcode & 1.133 & $0,65 \%$ & 378 & $0,46 \%$ \\
\hline Worms & 130 & $0,07 \%$ & 44 & $0,05 \%$ \\
\hline Total & 175.341 & $100,00 \%$ & 82.332 & $100,00 \%$ \\
\hline
\end{tabular}

effectively and reliably.

However, because the UNSW-NB15 dataset is still quite new, it has not been used by many scholars in their studies. Therefore, there are limitations when comparing results with other studies.

\subsection{Evaluation metrics}

We denote:

$T P_{i}$ : the number of correctly classified instances for class $c_{i}$

$F P_{i}$ : the number of instances that were incorrectly classified to the class $c_{i}$

$T N_{i}$ : the number of correctly classified instances that do not belong to the class $c_{i}$

$F N_{i}$ : the number of instances that were not classified as belonging to the class $c_{i}$

The performance evaluation of the classifiers is done by measuring and comparing metrics:

- Accuracy $_{i}=\left(T P_{i}+T N_{i}\right) /\left(T P_{i}+F P_{i}+T N_{i}+F N_{i}\right)$

- Sensitivity SeTP $_{i}=\left(T P_{i}\right) /\left(T P_{i}+F N_{i}\right)$

- Specificity S $_{i}=T N R_{i}=T N_{i} /\left(T N_{i}+F P_{i}\right)$

- Efficiency f $_{i}=\left(\right.$ Sensitivity $_{i}+$ Specificity $\left._{i}\right) / 2$

- Precision $_{i}=T P_{i} /\left(T P_{i}+F P_{i}\right)$

- $F N R_{i}=F N_{i} /\left(F N_{i}+T P_{i}\right)$

- $F P R_{i}=F P_{i} /\left(F P_{i}+T N_{i}\right)$

- Time for training and testing
The use of Accuracy to assess the quality of classification has been used by many scholars. However, the class distribution in most nonlinear classification problems is very imbalanced. Therefore, using Accuracy to evaluate the quality of classification of a model is not really effective. Therefore, the more comprehensive metrics recommended for the evaluation of $F$-Measure and $G$-Means are calculated as follows [12], [13]:

$$
F-\text { Measure }_{i}=\frac{\left(1+\beta^{2}\right) \times \text { Precision }_{i} \times \text { Recall }_{i}}{\beta^{2} \times \text { Precision }_{i}+\text { Recall }_{i}}
$$

Here, $\beta$ is the coefficient that adjusts the relationship between Precision and Recall and usually $\beta=1$. $F$-Measure shows the harmonious correlation between Precision and Recall. F-measure values are high when both Precision and Recall are high. And the $G-M e a n s$ indicator is calculated:

$$
G-\text { Means }_{i}=\sqrt{\text { Sensitivity }_{i} \times \text { Specificity }_{i}}
$$

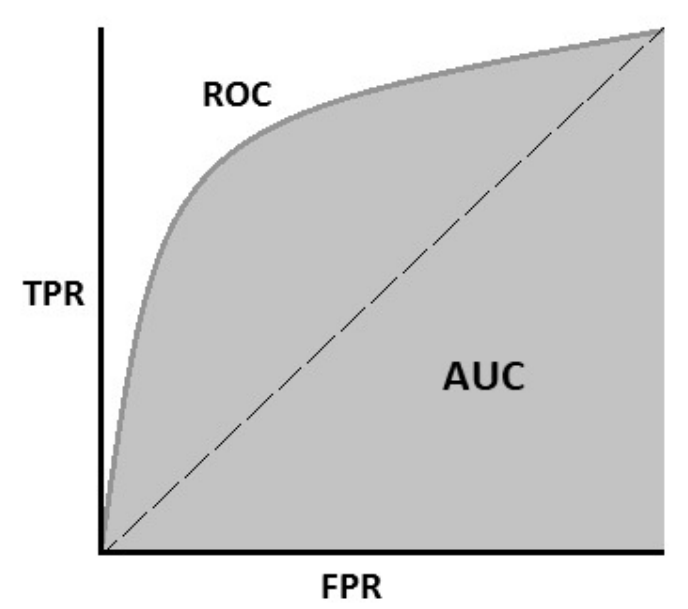

Figure 1. AUC - ROC Curve.

ROC (Receiver Operating Characteristics) is a method of calculating the performance of a classification model according to different classification thresholds. Assuming a binary classification problem (2 classes) using Logistic Regression, the selection of different classification thresholds [0..1] will affect the classification ability of the model and the level of influence of thresholds needs to be calculated. $R O C$ is a probability and Area Under The Curve $(A U C)$ representing the degree of classification of the model. Meaning of the interpretable $A U C$ : It is the probability that a randomly sampled positive sample will be ranked higher than a randomized negative sample, $A U C=P\left(\left(\operatorname{score}\left(x^{+}\right)>\operatorname{score}\left(x^{-}\right)\right)\right.$ The higher the $A U C$, the more accurate the model is in classifying classes. The ROC curve represents the pair 
of metrics (TPR, FPR) at each threshold with $T P R$ is the vertical axis and FPR is the horizontal axis (Fig. 1). The evaluation metrics used in the experiments of this paper are: Sensitivity, Specificity, Precision, FMeasure, $G-M e a n s, A U C$, Training time and Testing time.

\subsection{Using BFE combined with IG, GR and CA to reduce features}

Reduce features on the WORMS class. The results of using BFE in combination with IG, GR and CA on WORMS class with different machine learning algorithms are shown in the Table 3, Table 4 and Table 5. The machine learning techniques used for comparison include: DT, NB, LR, SVM, kNN and ANN. Accordingly, the best results are obtained when the feature reduction algorithm used is IG-BFE, the best machine learning algorithm used is DT, the number of remaining features after being reduced is 38 . This is because as shown in Table 3, Table 4 and Table 5: although the $G-$ Means of NB, LR and kNN are higher, but they have a lower Precision, due to a high false alarm rate (normal access is misclassified as an attack); In contrast, SVM has a high Precision, but has too low Sensitivity (attack access is misclassified as normal).

Table 3. Results of using IG-BFE algorithm on WORMS.

\begin{tabular}{|l|c|c|c|c|c|c|}
\hline Metrics & DT & NB & LR & SVM & kNN & ANN \\
\hline Sensitivity & 0.8385 & 0.8769 & 0.8615 & 0.1615 & 0.9077 & 0.8462 \\
\hline Specificity & 0.9992 & 0.9681 & 0.9884 & 1.0000 & 0.9982 & 0.9977 \\
\hline Precision & 0.6987 & 0.0600 & 0.1468 & 0.9545 & 0.5339 & 0.4622 \\
\hline F-Measure & 0.7622 & 0.1124 & 0.2508 & 0.2763 & 0.6724 & 0.5978 \\
\hline G-Means & $\mathbf{0 . 9 1 5 3}$ & 0.9214 & 0.9228 & 0.4019 & 0.9519 & 0.9188 \\
\hline AUC & 0.9489 & 0.9734 & 0.9726 & 0.5808 & 0.9519 & 0.9612 \\
\hline Train time & $2.88 \mathrm{~s}$ & $344 \mathrm{~ms}$ & $64 \mathrm{~s}$ & $32 \mathrm{~s}$ & $0 \mathrm{~ms}$ & $445 \mathrm{~s}$ \\
\hline Test time & $31 \mathrm{~ms}$ & $1.98 \mathrm{~s}$ & $156 \mathrm{~ms}$ & $25 \mathrm{~s}$ & $520 \mathrm{~s}$ & $266 \mathrm{~ms}$ \\
\hline Features & 38 & 32 & 37 & 41 & 33 & 42 \\
\hline
\end{tabular}

Table 4. Results of using GR-BFE algorithm on WORMS.

\begin{tabular}{|l|c|c|c|c|c|c|}
\hline Metrics & DT & NB & LR & SVM & kNN & ANN \\
\hline Sensitivity & 0.8385 & 0.8923 & 0.9231 & 0.1615 & 0.9231 & 0.8462 \\
\hline Specificity & 0.9991 & 0.9672 & 0.9543 & 1.0000 & 0.9981 & 0.9977 \\
\hline Precision & 0.6855 & 0.0593 & 0.0447 & 0.9545 & 0.5333 & 0.4622 \\
\hline F-Measure & 0.7543 & 0.1113 & 0.0853 & 0.2763 & 0.6761 & 0.5978 \\
\hline G-Means & 0.9153 & 0.9290 & 0.9385 & 0.4019 & 0.9599 & 0.9188 \\
\hline AUC & 0.9489 & 0.9730 & 0.9510 & 0.5808 & 0.9521 & 0.9612 \\
\hline Train time & $2.95 \mathrm{~s}$ & $266 \mathrm{~ms}$ & $616 \mathrm{~s}$ & $43 \mathrm{~s}$ & $6 \mathrm{~ms}$ & $265 \mathrm{~s}$ \\
\hline Test time & $44 \mathrm{~ms}$ & $1 \mathrm{~s}$ & $125 \mathrm{~ms}$ & $17 \mathrm{~s}$ & $414 \mathrm{~s}$ & $177 \mathrm{~ms}$ \\
\hline Features & 39 & 30 & 39 & 41 & 34 & 42 \\
\hline
\end{tabular}

Table 6 compares the results of using IG-BFE with other feature reduction algorithms such as: Recursive Feature Elimination (RFE) algorithm combined with the ranking of the features using: Entropy, Gini index (Gini), Ridge, Random Forest and the average value of the above indexes (Avg). Thereby, IG-BFE gives better results when the features are not reduced. Although
Table 5. Results of using CA-BFE algorithm on WORMS.

\begin{tabular}{|l|c|c|c|c|c|c|}
\hline Metrics & DT & NB & LR & SVM & kNN & ANN \\
\hline Sensitivity & 0.8385 & 0.8615 & 0.9077 & 0.1615 & 0.9077 & 0.8462 \\
\hline Specificity & 0.9991 & 0.9700 & 0.9723 & 1.0000 & 0.9981 & 0.9977 \\
\hline Precision & 0.6855 & 0.0625 & 0.0707 & 0.9545 & 0.5291 & 0.4622 \\
\hline F-Measure & 0.7543 & 0.1165 & 0.1311 & 0.2763 & 0.6686 & 0.5978 \\
\hline G-Means & 0.9153 & 0.9142 & 0.9394 & 0.4019 & 0.9518 & 0.9188 \\
\hline AUC & 0.9489 & 0.9742 & 0.9621 & 0.5808 & 0.9419 & 0.9612 \\
\hline Train time & $2.25 \mathrm{~s}$ & $367 \mathrm{~ms}$ & $245 \mathrm{~s}$ & $43 \mathrm{~s}$ & $14 \mathrm{~ms}$ & $266 \mathrm{~s}$ \\
\hline Test time & $86 \mathrm{~ms}$ & $1.16 \mathrm{~s}$ & $133 \mathrm{~ms}$ & $16 \mathrm{~s}$ & $528 \mathrm{~s}$ & $170 \mathrm{~ms}$ \\
\hline Features & 39 & 31 & 36 & 41 & 35 & 42 \\
\hline
\end{tabular}

G-Means of other methods are better than IG-BFE, but the false alarm rate also increases, so IG-BFE is still selected.

Table 6. Compare IG-BFE with other algorithms on WORMS.

\begin{tabular}{|l|c|c|c|c|c|c|}
\hline & Origin & BFE & Entropy & Gini & Ridge & Avg \\
\hline Sensitivity & 0.8231 & 0.8385 & 0.8769 & 0.8692 & 0.8846 & 0.9462 \\
\hline Specificity & 0.9991 & 0.9992 & 0.9981 & 0.9983 & 0.9982 & 0.9659 \\
\hline Precision & 0.6903 & 0.6987 & 0.5182 & 0.5407 & 0.5374 & 0.0605 \\
\hline F-Measure & 0.7509 & 0.7622 & 0.6514 & 0.6667 & 0.6686 & 0.1137 \\
\hline G-Means & 0.9068 & $\mathbf{0 . 9 1 5 3}$ & 0.9356 & 0.9315 & 0.9397 & 0.9560 \\
\hline AUC & 0.9448 & 0.9489 & 0.9339 & 0.9303 & 0.9558 & 0.9942 \\
\hline Train time & $3.84 \mathrm{~s}$ & $2.88 \mathrm{~s}$ & $6 \mathrm{~ms}$ & $6 \mathrm{~ms}$ & $5 \mathrm{~ms}$ & $475 \mathrm{~s}$ \\
\hline Test time & $48 \mathrm{~ms}$ & $31 \mathrm{~ms}$ & $621 \mathrm{~s}$ & $561 \mathrm{~s}$ & $581 \mathrm{~s}$ & $297 \mathrm{~ms}$ \\
\hline Features & 42 & 38 & 38 & 40 & 17 & 17 \\
\hline ML & DT & DT & kNN & kNN & kNN & ANN \\
\hline
\end{tabular}

Reduce features on the other classes. Similarly, the results of using BFE on SHELLCODE class are shown in the Table 7, Table 8 and Table 9. Accordingly, the best results are obtained when the feature reduction algorithm used is CA-BFE, the best machine learning algorithm used is DT, the number of remaining features after being reduced is 32 . Table 10 compares the results of using CA-BFE with other algorithms. Thereby, CABFE gives better results all of rest algorithms.

Table 7. Results of using IG-BFE algorithm on SHELLCODE.

\begin{tabular}{|l|c|c|c|c|c|c|}
\hline Metrics & DT & NB & LR & SVM & kNN & ANN \\
\hline Sensitivity & 0.9029 & 0.9894 & 0.9815 & 0 & 0.6920 & 0.9320 \\
\hline Specificity & 0.9919 & 0.9185 & 0.9388 & 0 & 0.9985 & 0.9800 \\
\hline Precision & 0.6926 & 0.1973 & 0.2449 & 0 & 0.9032 & 0.4853 \\
\hline F-Measure & 0.7839 & 0.3289 & 0.3920 & 0 & 0.7836 & 0.6383 \\
\hline G-Means & 0.9464 & 0.9533 & 0.9599 & 0 & 0.8312 & 0.9557 \\
\hline AUC & 0.9556 & 0.9661 & 0.9753 & 0 & 0.8923 & 0.9813 \\
\hline Train time & $2.2 \mathrm{~s}$ & $297 \mathrm{~ms}$ & $28 \mathrm{~s}$ & 0 & $16 \mathrm{~ms}$ & $361 \mathrm{~s}$ \\
\hline Test time & $47 \mathrm{~ms}$ & $2 \mathrm{~s}$ & $172 \mathrm{~ms}$ & 0 & $508 \mathrm{~s}$ & $203 \mathrm{~ms}$ \\
\hline Features & 33 & 27 & 39 & 0 & 30 & 32 \\
\hline
\end{tabular}

The results of using BFE in combination with IG, GR and CA on BACKDOOR class with different machine learning algorithms are shown in the Table 11, Table 12 and Table 13. Accordingly, the best results are obtained when the feature reduction algorithm used is CA-BFE, the best machine learning algorithm used is DT, the number of remaining features after being reduced is 34. Table 14 compares the results of using CA-BFE with 
Table 8. Results of using GR-BFE algorithm on SHELLCODE.

\begin{tabular}{|l|c|c|c|c|c|c|}
\hline Metrics & DT & NB & LR & SVM & kNN & ANN \\
\hline Sensitivity & 0.9091 & 0.9912 & 0.9885 & 0 & 0.9303 & 0.9056 \\
\hline Specificity & 0.9929 & 0.9158 & 0.9060 & 0 & 0.9114 & 0.9803 \\
\hline Precision & 0.7203 & 0.1924 & 0.1755 & 0 & 0.1752 & 0.4817 \\
\hline F-Measure & 0.8037 & 0.3222 & 0.2981 & 0 & 0.2948 & 0.6289 \\
\hline G-Means & 0.9501 & 0.9528 & 0.9464 & 0 & 0.9208 & 0.9422 \\
\hline AUC & 0.9747 & 0.9650 & 0.9775 & 0 & 0.9497 & 0.9759 \\
\hline Train time & $3.1 \mathrm{~s}$ & $244 \mathrm{~ms}$ & $32 \mathrm{~s}$ & 0 & $5 \mathrm{~ms}$ & $249 \mathrm{~s}$ \\
\hline Test time & $55 \mathrm{~ms}$ & $896 \mathrm{~ms}$ & $122 \mathrm{~ms}$ & 0 & $464 \mathrm{~s}$ & $159 \mathrm{~ms}$ \\
\hline Features & 34 & 26 & 40 & 0 & 33 & 37 \\
\hline
\end{tabular}

Table 9. Results of using CA-BFE algorithm on SHELLCODE.

\begin{tabular}{|l|c|c|c|c|c|c|}
\hline Metrics & DT & NB & LR & SVM & kNN & ANN \\
\hline Sensitivity & 0.9276 & 0.9550 & 0.9956 & 0 & 0.9276 & 0.9267 \\
\hline Specificity & 0.9925 & 0.9548 & 0.8480 & 0 & 0.9045 & 0.9747 \\
\hline Precision & 0.7145 & 0.2996 & 0.1170 & 0 & 0.1642 & 0.4258 \\
\hline F-Measure & 0.8072 & 0.4562 & 0.2094 & 0 & 0.2790 & 0.5835 \\
\hline G-Means & $\mathbf{0 . 9 5 9 5}$ & 0.9549 & 0.9188 & 0 & 0.9160 & 0.9504 \\
\hline AUC & 0.9763 & 0.9837 & 0.9717 & 0 & 0.9415 & 0.9790 \\
\hline Train time & $2.58 \mathrm{~s}$ & $296 \mathrm{~ms}$ & $35 \mathrm{~s}$ & 0 & $6 \mathrm{~ms}$ & $223 \mathrm{~s}$ \\
\hline Test time & $43 \mathrm{~ms}$ & $1.17 \mathrm{~s}$ & $123 \mathrm{~ms}$ & 0 & $501 \mathrm{~s}$ & $149 \mathrm{~ms}$ \\
\hline Features & 32 & 32 & 39 & 0 & 32 & 33 \\
\hline
\end{tabular}

Table 10. Compare CA-BFE with other algorithms on SHELLCODE.

\begin{tabular}{|l|c|c|c|c|c|c|}
\hline & Origin & BFE & Entropy & Gini & Ridge & Avg \\
\hline Sensitivity & 0.8244 & 0.9276 & 0.9356 & 0.9356 & 0.9285 & 0.8782 \\
\hline Specificity & 0.9942 & 0.9925 & 0.9089 & 0.9093 & 0.9336 & 0.9934 \\
\hline Precision & 0.7407 & 0.7145 & 0.1720 & 0.1726 & 0.2204 & 0.7284 \\
\hline F-Measure & 0.7803 & 0.8072 & 0.2906 & 0.2914 & 0.3562 & 0.7963 \\
\hline G-Means & 0.9053 & $\mathbf{0 . 9 5 9 5}$ & 0.9221 & 0.9223 & 0.9310 & 0.9340 \\
\hline AUC & 0.9270 & 0.9763 & 0.9738 & 0.9770 & 0.9786 & 0.9600 \\
\hline Train time & $3.97 \mathrm{~s}$ & $2.58 \mathrm{~s}$ & $320 \mathrm{~ms}$ & $344 \mathrm{~ms}$ & $383 \mathrm{~ms}$ & $3.93 \mathrm{~s}$ \\
\hline Test time & $48 \mathrm{~ms}$ & $43 \mathrm{~ms}$ & $1.38 \mathrm{~s}$ & $1.35 \mathrm{~s}$ & $1.32 \mathrm{~s}$ & $62 \mathrm{~ms}$ \\
\hline Features & 42 & 32 & 17 & 15 & 15 & 24 \\
\hline ML & $\mathrm{DT}$ & $\mathrm{DT}$ & $\mathrm{NB}$ & $\mathrm{NB}$ & $\mathrm{NB}$ & $\mathrm{DT}$ \\
\hline
\end{tabular}

Table 11. Results of using IG-BFE algorithm on BACKDOOR.

\begin{tabular}{|l|c|c|c|c|c|c|}
\hline Metrics & DT & NB & LR & SVM & kNN & ANN \\
\hline Sensitivity & 0.9651 & 0.8814 & 0.8952 & 0.3328 & 0.9152 & 0.8213 \\
\hline Specificity & 0.9932 & 0.9134 & 0.9743 & 0.9993 & 0.9735 & 0.9763 \\
\hline Precision & 0.8160 & 0.2409 & 0.5210 & 0.9356 & 0.5187 & 0.5192 \\
\hline F-Measure & 0.8843 & 0.3784 & 0.6587 & 0.4909 & 0.6621 & 0.6362 \\
\hline G-Means & 0.9790 & 0.8973 & 0.9339 & 0.5766 & 0.9439 & 0.8954 \\
\hline AUC & 0.9796 & 0.9610 & 0.9825 & 0.6660 & 0.9563 & 0.9424 \\
\hline Train time & $4.47 \mathrm{~s}$ & $313 \mathrm{~ms}$ & $5.78 \mathrm{~s}$ & $116 \mathrm{~s}$ & $16 \mathrm{~ms}$ & $423 \mathrm{~s}$ \\
\hline Test time & $46 \mathrm{~ms}$ & $1.45 \mathrm{~s}$ & $109 \mathrm{~ms}$ & $106 \mathrm{~s}$ & $484 \mathrm{~s}$ & $203 \mathrm{~ms}$ \\
\hline Features & 33 & 28 & 24 & 14 & 26 & 34 \\
\hline
\end{tabular}

Table 12. Results of using GR-BFE algorithm on BACKDOOR.

\begin{tabular}{|l|c|c|c|c|c|c|}
\hline Metrics & DT & NB & LR & SVM & kNN & ANN \\
\hline Sensitivity & 0.9668 & 0.8969 & 0.9152 & 0.3408 & 0.9559 & 0.8786 \\
\hline Specificity & 0.9931 & 0.8988 & 0.8952 & 0.9996 & 0.9895 & 0.9906 \\
\hline Precision & 0.8127 & 0.2165 & 0.2141 & 0.9597 & 0.7401 & 0.7439 \\
\hline F-Measure & 0.8831 & 0.3489 & 0.3470 & 0.5030 & 0.8343 & 0.8057 \\
\hline G-Means & 0.9798 & 0.8979 & 0.9052 & 0.5836 & 0.9726 & 0.9329 \\
\hline AUC & 0.9805 & 0.9551 & 0.9016 & 0.6702 & 0.9841 & 0.9775 \\
\hline Train time & $3.26 \mathrm{~s}$ & $233 \mathrm{~ms}$ & $4.18 \mathrm{~s}$ & $92 \mathrm{~s}$ & $6 \mathrm{~ms}$ & $213 \mathrm{~s}$ \\
\hline Test time & $53 \mathrm{~ms}$ & $806 \mathrm{~ms}$ & $76 \mathrm{~ms}$ & $31 \mathrm{~s}$ & $465 \mathrm{~s}$ & $130 \mathrm{~ms}$ \\
\hline Features & 35 & 29 & 26 & 12 & 28 & 29 \\
\hline
\end{tabular}

The results of using BFE on RECONNAISSANCE class other algorithms. are shown in the Table 19, Table 20 and Table 21. The best feature reduction algorithm used is CA-BFE, the best machine learning algorithm used is DT, the number of remaining features after being reduced is 35. Table 22 compares the results of using CA-BFE with

The results of using BFE on DOS class are shown in the Table 23, Table 24 and Table 25. The best feature

Table 13. Results of using CA-BFE algorithm on BACKDOOR.

\begin{tabular}{|l|c|c|c|c|c|c|}
\hline Metrics & DT & NB & LR & SVM & kNN & ANN \\
\hline Sensitivity & 0.9668 & 0.9049 & 0.8666 & 0.3402 & 0.8356 & 0.9078 \\
\hline Specificity & 0.9931 & 0.8998 & 0.8099 & 0.9991 & 0.9954 & 0.9770 \\
\hline Precision & 0.8139 & 0.2197 & 0.1245 & 0.9195 & 0.8497 & 0.5515 \\
\hline F-Measure & 0.8838 & 0.3535 & 0.2177 & 0.4967 & 0.8426 & 0.6861 \\
\hline G-Means & $\mathbf{0 . 9 7 9 9}$ & 0.9024 & 0.8378 & 0.5830 & 0.9120 & 0.9418 \\
\hline AUC & 0.9808 & 0.9588 & 0.8293 & 0.6696 & 0.9377 & 0.9754 \\
\hline Train time & $3.07 \mathrm{~s}$ & $265 \mathrm{~ms}$ & $4.37 \mathrm{~s}$ & $67 \mathrm{~s}$ & $7 \mathrm{~ms}$ & $199 \mathrm{~s}$ \\
\hline Test time & $53 \mathrm{~ms}$ & $882 \mathrm{~ms}$ & $85 \mathrm{~ms}$ & $27 \mathrm{~s}$ & $455 \mathrm{~s}$ & $114 \mathrm{~ms}$ \\
\hline Features & 34 & 30 & 26 & 11 & 30 & 27 \\
\hline
\end{tabular}

other algorithms. Thereby, CA-BFE gives better results all of rest algorithms.

Table 14. Compare CA-BFE with other algorithms on BACKDOOR.

\begin{tabular}{|l|c|c|c|c|c|c|}
\hline & Origin & BFE & Entropy & Gini & Ridge & Avg \\
\hline Sensitivity & 0.9416 & 0.9668 & 0.9548 & 0.9548 & 0.9416 & 0.9611 \\
\hline Specificity & 0.9894 & 0.9931 & 0.9892 & 0.9892 & 0.9894 & 0.9844 \\
\hline Precision & 0.7339 & 0.8139 & 0.7340 & 0.7340 & 0.7339 & 0.6580 \\
\hline F-Measure & 0.8249 & 0.8838 & 0.8300 & 0.8300 & 0.8249 & 0.7812 \\
\hline G-Means & 0.9652 & $\mathbf{0 . 9 7 9 9}$ & 0.9718 & 0.9718 & 0.9652 & 0.9727 \\
\hline AUC & 0.9611 & 0.9808 & 0.9689 & 0.9689 & 0.9611 & 0.9717 \\
\hline Train time & $8.73 \mathrm{~s}$ & $3.07 \mathrm{~s}$ & $6.97 \mathrm{~s}$ & $7.12 \mathrm{~s}$ & $7.34 \mathrm{~s}$ & $7.31 \mathrm{~s}$ \\
\hline Test time & $78 \mathrm{~ms}$ & $53 \mathrm{~ms}$ & $61 \mathrm{~ms}$ & $67 \mathrm{~ms}$ & $75 \mathrm{~ms}$ & $62 \mathrm{~ms}$ \\
\hline Features & 42 & 34 & 30 & 31 & 41 & 28 \\
\hline ML & DT & DT & DT & DT & DT & DT \\
\hline
\end{tabular}

Similarly, the results of using BFE on ANALYSIS class are shown in the Table 15, Table 16 and Table 17. Accordingly, the best results are obtained when the feature reduction algorithm used is IG-BFE, the best machine learning algorithm used is $\mathrm{kNN}$, the number of remaining features after being reduced is 23 . Table 18 compares the results of using IG-BFE with other algorithms. Thereby, IG-BFE gives better results all of rest algorithms.

Table 15. Results of using IG-BFE algorithm on ANALYSIS.

\begin{tabular}{|l|c|c|c|c|c|c|}
\hline Metrics & DT & NB & LR & SVM & kNN & ANN \\
\hline Sensitivity & 0.8590 & 0.7570 & 0.7515 & 0.2460 & 0.9055 & 0.6985 \\
\hline Specificity & 0.9748 & 0.9603 & 0.8190 & 0.9991 & 0.9805 & 0.9939 \\
\hline Precision & 0.5492 & 0.4050 & 0.1292 & 0.9044 & 0.6234 & 0.8033 \\
\hline F-Measure & 0.6700 & 0.5277 & 0.2204 & 0.3868 & 0.7384 & 0.7473 \\
\hline G-Means & 0.9151 & 0.8526 & 0.7845 & 0.4958 & $\mathbf{0 . 9 4 2 2}$ & 0.8332 \\
\hline AUC & 0.9169 & 0.9502 & 0.8394 & 0.6225 & 0.9338 & 0.8019 \\
\hline Train time & $5.80 \mathrm{~s}$ & $219 \mathrm{~ms}$ & $34 \mathrm{~s}$ & $52 \mathrm{~s}$ & $0 \mathrm{~ms}$ & $435 \mathrm{~s}$ \\
\hline Test time & $47 \mathrm{~ms}$ & $1.34 \mathrm{~s}$ & $141 \mathrm{~ms}$ & $45 \mathrm{~s}$ & $408 \mathrm{~s}$ & $203 \mathrm{~ms}$ \\
\hline Features & 41 & 20 & 39 & 16 & 23 & 36 \\
\hline
\end{tabular}


Table 16. Results of using GR-BFE algorithm on ANALYSIS

\begin{tabular}{|l|c|c|c|c|c|c|}
\hline Metrics & DT & NB & LR & SVM & kNN & ANN \\
\hline Sensitivity & 0.8590 & 0.7440 & 0.9040 & 0.2715 & 0.8620 & 0.6420 \\
\hline Specificity & 0.9748 & 0.9759 & 0.8099 & 0.9970 & 0.9778 & 0.9994 \\
\hline Precision & 0.5492 & 0.5239 & 0.1452 & 0.7648 & 0.5809 & 0.9764 \\
\hline F-Measure & 0.6700 & 0.6149 & 0.2502 & 0.4007 & 0.6940 & 0.7747 \\
\hline G-Means & 0.9151 & 0.8521 & 0.8557 & 0.5203 & 0.9181 & 0.8010 \\
\hline AUC & 0.9169 & 0.9631 & 0.8421 & 0.6343 & 0.9008 & 0.9078 \\
\hline Train time & $2.59 \mathrm{~s}$ & $193 \mathrm{~ms}$ & $17.5 \mathrm{~s}$ & $32 \mathrm{~s}$ & $7 \mathrm{~ms}$ & $255 \mathrm{~s}$ \\
\hline Test time & $41 \mathrm{~ms}$ & $612 \mathrm{~ms}$ & $101 \mathrm{~ms}$ & $17.6 \mathrm{~s}$ & $458 \mathrm{~s}$ & $152 \mathrm{~ms}$ \\
\hline Features & 41 & 18 & 34 & 10 & 28 & 38 \\
\hline
\end{tabular}

Table 17. Results of using CA-BFE algorithm on ANALYSIS.

\begin{tabular}{|l|c|c|c|c|c|c|}
\hline Metrics & DT & NB & LR & SVM & kNN & ANN \\
\hline Sensitivity & 0.8590 & 0.7325 & 0.8660 & 0.2500 & 0.8385 & 0.6480 \\
\hline Specificity & 0.9748 & 0.9868 & 0.8146 & 0.9996 & 0.9778 & 0.9987 \\
\hline Precision & 0.5492 & 0.6653 & 0.1430 & 0.9597 & 0.5739 & 0.9453 \\
\hline F-Measure & 0.6700 & 0.6973 & 0.2454 & 0.3967 & 0.6814 & 0.7689 \\
\hline G-Means & 0.9151 & 0.8502 & 0.8399 & 0.4999 & 0.9055 & 0.8044 \\
\hline AUC & 0.9169 & 0.9647 & 0.8411 & 0.6248 & 0.8916 & 0.8639 \\
\hline Train time & $2.86 \mathrm{~s}$ & $191 \mathrm{~ms}$ & $22 \mathrm{~s}$ & $67.6 \mathrm{~s}$ & $9 \mathrm{~ms}$ & $254 \mathrm{~s}$ \\
\hline Test time & $45 \mathrm{~ms}$ & $673 \mathrm{~ms}$ & $105 \mathrm{~ms}$ & $27.1 \mathrm{~s}$ & $487 \mathrm{~s}$ & $164 \mathrm{~ms}$ \\
\hline Features & 41 & 21 & 31 & 16 & 32 & 38 \\
\hline
\end{tabular}

Table 18. Compare IG-BFE with other algorithms on ANALYSIS.

\begin{tabular}{|l|c|c|c|c|c|c|}
\hline & Origin & BFE & Entropy & Gini & Ridge & Avg \\
\hline Sensitivity & 0.8570 & 0.9055 & 0.8585 & 0.8590 & 0.8570 & 0.8605 \\
\hline Specificity & 0.9743 & 0.9805 & 0.9757 & 0.9748 & 0.9743 & 0.9762 \\
\hline Precision & 0.5440 & 0.6234 & 0.5580 & 0.5492 & 0.5440 & 0.5633 \\
\hline F-Measure & 0.6655 & 0.7384 & 0.6764 & 0.6700 & 0.6655 & 0.6809 \\
\hline G-Means & 0.9138 & $\mathbf{0 . 9 4 2 2}$ & 0.9152 & 0.9151 & 0.9138 & 0.9165 \\
\hline AUC & 0.8670 & 0.9338 & 0.8650 & 0.9169 & 0.8670 & 0.9146 \\
\hline Train time & $6.3 \mathrm{~s}$ & $0 \mathrm{~ms}$ & $4.65 \mathrm{~s}$ & $5.17 \mathrm{~s}$ & $5.56 \mathrm{~s}$ & $6.36 \mathrm{~s}$ \\
\hline Test time & $102 \mathrm{~ms}$ & $408.2 \mathrm{~s}$ & $54 \mathrm{~ms}$ & $60 \mathrm{~ms}$ & $64 \mathrm{~ms}$ & $47 \mathrm{~ms}$ \\
\hline Features & 42 & 23 & 17 & 36 & 40 & 10 \\
\hline ML & DT & kNN & DT & DT & DT & DT \\
\hline
\end{tabular}

Table 19. Results of using IG-BFE on RECONNAISSANCE.

\begin{tabular}{|l|c|c|c|c|c|c|}
\hline Metrics & DT & NB & LR & SVM & kNN & ANN \\
\hline Sensitivity & 0.9529 & 0.9736 & 0.9852 & 0.9552 & 0.9774 & 0.9604 \\
\hline Specificity & 0.9931 & 0.8634 & 0.9441 & 0.9310 & 0.8935 & 0.9841 \\
\hline Precision & 0.9629 & 0.5718 & 0.7675 & 0.7218 & 0.6322 & 0.9186 \\
\hline F-Measure & 0.9579 & 0.7205 & 0.8628 & 0.8222 & 0.7678 & 0.9390 \\
\hline G-Means & 0.9728 & 0.9169 & 0.9644 & 0.9430 & 0.9345 & 0.9722 \\
\hline AUC & 0.9867 & 0.9507 & 0.9908 & 0.9431 & 0.9419 & 0.9891 \\
\hline Train time & $5.28 \mathrm{~s}$ & $500 \mathrm{~ms}$ & $16.3 \mathrm{~s}$ & $205.8 \mathrm{~s}$ & $0 \mathrm{~ms}$ & $441.4 \mathrm{~s}$ \\
\hline Test time & $62 \mathrm{~ms}$ & $2.30 \mathrm{~s}$ & $141 \mathrm{~ms}$ & $285.5 \mathrm{~s}$ & $672.8 \mathrm{~s}$ & $250 \mathrm{~ms}$ \\
\hline Features & 33 & 31 & 32 & 17 & 32 & 33 \\
\hline
\end{tabular}

Table 20. Results of using GR-BFE on RECONNAISSANCE.

\begin{tabular}{|l|c|c|c|c|c|c|}
\hline Metrics & DT & NB & LR & SVM & kNN & ANN \\
\hline Sensitivity & 0.9705 & 0.9776 & 0.9672 & 0.9358 & 0.8809 & 0.9637 \\
\hline Specificity & 0.9900 & 0.8709 & 0.9442 & 0.9404 & 0.9942 & 0.9803 \\
\hline Precision & 0.9477 & 0.5866 & 0.7646 & 0.7463 & 0.9662 & 0.9018 \\
\hline F-Measure & 0.9589 & 0.7332 & 0.8541 & 0.8303 & 0.9216 & 0.9317 \\
\hline G-Means & 0.9802 & 0.9227 & 0.9556 & 0.9381 & 0.9359 & 0.9720 \\
\hline AUC & 0.9871 & 0.9510 & 0.9893 & 0.9381 & 0.9643 & 0.9900 \\
\hline Train time & $3.49 \mathrm{~s}$ & $249 \mathrm{~ms}$ & $14.93 \mathrm{~s}$ & $391 \mathrm{~s}$ & $21 \mathrm{~ms}$ & $273 \mathrm{~s}$ \\
\hline Test time & $52 \mathrm{~ms}$ & $846 \mathrm{~ms}$ & $112 \mathrm{~ms}$ & $453 \mathrm{~s}$ & $687 \mathrm{~s}$ & $206 \mathrm{~ms}$ \\
\hline Features & 36 & 22 & 36 & 21 & 32 & 35 \\
\hline
\end{tabular}

reduction algorithm used is GR-BFE, the best machine learning algorithm used is DT, the number of remaining
Table 21. Results of using CA-BFE on RECONNAISSANCE.

\begin{tabular}{|l|c|c|c|c|c|c|}
\hline Metrics & DT & NB & LR & SVM & kNN & ANN \\
\hline Sensitivity & 0.9705 & 0.9748 & 0.9710 & 0.9343 & 0.9750 & 0.9617 \\
\hline Specificity & 0.9900 & 0.8642 & 0.9577 & 0.9467 & 0.8993 & 0.9869 \\
\hline Precision & 0.9477 & 0.5736 & 0.8113 & 0.7666 & 0.6446 & 0.9321 \\
\hline F-Measure & 0.9589 & 0.7222 & 0.8840 & 0.8422 & 0.7761 & 0.9467 \\
\hline G-Means & $\mathbf{0 . 9 8 0 2}$ & 0.9179 & 0.9643 & 0.9405 & 0.9364 & 0.9742 \\
\hline AUC & 0.9871 & 0.9508 & 0.9904 & 0.9405 & 0.9515 & 0.9910 \\
\hline Train time & $4.26 \mathrm{~s}$ & $417 \mathrm{~ms}$ & $8.67 \mathrm{~s}$ & $415.1 \mathrm{~s}$ & $16 \mathrm{~ms}$ & $285.1 \mathrm{~s}$ \\
\hline Test time & $41 \mathrm{~ms}$ & $1.21 \mathrm{~s}$ & $110 \mathrm{~ms}$ & $441 \mathrm{~s}$ & $709 \mathrm{~s}$ & $220 \mathrm{~ms}$ \\
\hline Features & 35 & 30 & 30 & 21 & 33 & 37 \\
\hline
\end{tabular}

Table 22. Compare CA-BFE with other algorithms on RECONNAISSANCE.

\begin{tabular}{|l|c|c|c|c|c|c|}
\hline & Origin & BFE & Entropy & Gini & Ridge & Avg \\
\hline Sensitivity & 0.9172 & 0.9705 & 0.9435 & 0.9435 & 0.9562 & 0.9720 \\
\hline Specificity & 0.9933 & 0.9900 & 0.9863 & 0.9863 & 0.9698 & 0.9738 \\
\hline Precision & 0.9623 & 0.9477 & 0.9280 & 0.9280 & 0.8555 & 0.8741 \\
\hline F-Measure & 0.9392 & 0.9589 & 0.9357 & 0.9357 & 0.9031 & 0.9204 \\
\hline G-Means & 0.9545 & $\mathbf{0 . 9 8 0 2}$ & 0.9646 & 0.9646 & 0.9630 & 0.9729 \\
\hline AUC & 0.9583 & 0.9871 & 0.9915 & 0.9915 & 0.9843 & 0.9903 \\
\hline Train time & $7.65 \mathrm{~s}$ & $4.26 \mathrm{~s}$ & $314 \mathrm{~s}$ & $315 \mathrm{~s}$ & $293 \mathrm{~s}$ & $567 \mathrm{~s}$ \\
\hline Test time & $61 \mathrm{~ms}$ & $41 \mathrm{~ms}$ & $198 \mathrm{~ms}$ & $209 \mathrm{~ms}$ & $197 \mathrm{~ms}$ & $250 \mathrm{~ms}$ \\
\hline Features & 42 & 35 & 37 & 37 & 25 & 26 \\
\hline ML & DT & DT & ANN & ANN & ANN & ANN \\
\hline
\end{tabular}

features after being reduced is 30 .

Table 26 compares the results of using GR-BFE with other algorithms. Thereby, GR-BFE gives the best results.

Table 23. Results of using IG-BFE algorithm on DOS.

\begin{tabular}{|l|c|c|c|c|c|c|}
\hline Metrics & DT & NB & LR & SVM & kNN & ANN \\
\hline Sensitivity & 0.9689 & 0.8277 & 0.9336 & 0.8841 & 0.9561 & 0.9362 \\
\hline Specificity & 0.9890 & 0.9287 & 0.8977 & 0.9652 & 0.9822 & 0.9892 \\
\hline Precision & 0.9508 & 0.7176 & 0.6666 & 0.8478 & 0.9217 & 0.9498 \\
\hline F-Measure & 0.9598 & 0.7688 & 0.7777 & 0.8655 & 0.9386 & 0.9430 \\
\hline G-Means & 0.9789 & 0.8767 & 0.9155 & 0.9238 & 0.9691 & 0.9623 \\
\hline AUC & 0.9836 & 0.9045 & 0.9650 & 0.9246 & 0.9805 & 0.9887 \\
\hline Train time & $7.3 \mathrm{~s}$ & $420 \mathrm{~ms}$ & $9.97 \mathrm{~s}$ & $216 \mathrm{~s}$ & $0 \mathrm{~ms}$ & $535 \mathrm{~s}$ \\
\hline Test time & $62 \mathrm{~ms}$ & $1.69 \mathrm{~s}$ & $141 \mathrm{~ms}$ & $300 \mathrm{~s}$ & $602 \mathrm{~s}$ & $266 \mathrm{~ms}$ \\
\hline Features & 33 & 31 & 31 & 22 & 28 & 35 \\
\hline
\end{tabular}

Table 24. Results of using GR-BFE algorithm on DOS.

\begin{tabular}{|l|c|c|c|c|c|c|}
\hline Metrics & DT & NB & LR & SVM & kNN & ANN \\
\hline Sensitivity & 0.9690 & 0.8851 & 0.8166 & 0.8881 & 0.9641 & 0.9643 \\
\hline Specificity & 0.9903 & 0.8928 & 0.9823 & 0.9652 & 0.9817 & 0.9556 \\
\hline Precision & 0.9562 & 0.6439 & 0.9101 & 0.8484 & 0.9202 & 0.8261 \\
\hline F-Measure & 0.9626 & 0.7455 & 0.8608 & 0.8678 & 0.9416 & 0.8899 \\
\hline G-Means & $\mathbf{0 . 9 7 9 6}$ & 0.8890 & 0.8957 & 0.9259 & 0.9729 & 0.9599 \\
\hline AUC & 0.9799 & 0.9340 & 0.9820 & 0.9267 & 0.9843 & 0.9861 \\
\hline Train time & $4.06 \mathrm{~s}$ & $253 \mathrm{~ms}$ & $6.35 \mathrm{~s}$ & $305 \mathrm{~s}$ & $10 \mathrm{~ms}$ & $276 \mathrm{~s}$ \\
\hline Test time & $53 \mathrm{~ms}$ & $825 \mathrm{~ms}$ & $206 \mathrm{~ms}$ & $185 \mathrm{~s}$ & $629 \mathrm{~s}$ & $165 \mathrm{~ms}$ \\
\hline Features & 30 & 26 & 29 & 24 & 28 & 34 \\
\hline
\end{tabular}

The results of using BFE on FUZZERS class are shown in the Table 27, Table 28 and Table 29. The best feature reduction algorithm used is GR-BFE, the best machine learning algorithm used is SVM, the number of remaining features after being reduced is 15 . Table 30 compares the results of using GR-BFE with other 
Table 25. Results of using CA-BFE algorithm on DOS.

\begin{tabular}{|l|c|c|c|c|c|c|}
\hline Metrics & DT & NB & LR & SVM & kNN & ANN \\
\hline Sensitivity & 0.9724 & 0.8651 & 0.8541 & 0.8780 & 0.9641 & 0.9510 \\
\hline Specificity & 0.9874 & 0.9112 & 0.9802 & 0.9678 & 0.9643 & 0.9606 \\
\hline Precision & 0.9441 & 0.6809 & 0.9043 & 0.8567 & 0.8553 & 0.8411 \\
\hline F-Measure & 0.9580 & 0.7620 & 0.8785 & 0.8672 & 0.9064 & 0.8927 \\
\hline G-Means & 0.9798 & 0.8878 & 0.9150 & 0.9218 & 0.9642 & 0.9558 \\
\hline AUC & 0.9828 & 0.9259 & 0.9769 & 0.9229 & 0.9810 & 0.9838 \\
\hline Train time & $6.27 \mathrm{~s}$ & $304 \mathrm{~ms}$ & $5.67 \mathrm{~s}$ & $363 \mathrm{~s}$ & $17 \mathrm{~ms}$ & $283 \mathrm{~s}$ \\
\hline Test time & $67 \mathrm{~ms}$ & $917 \mathrm{~ms}$ & $105 \mathrm{~ms}$ & $419 \mathrm{~s}$ & $641 \mathrm{~s}$ & $180 \mathrm{~ms}$ \\
\hline Features & 34 & 28 & 30 & 31 & 28 & 35 \\
\hline
\end{tabular}

Table 26. Compare GR-BFE with other algorithms on DOS.

\begin{tabular}{|l|c|c|c|c|c|c|}
\hline & Origin & BFE & Entropy & Gini & Ridge & Avg \\
\hline Sensitivity & 0.9644 & 0.9690 & 0.9649 & 0.9649 & 0.9649 & 0.9644 \\
\hline Specificity & 0.9892 & 0.9903 & 0.9891 & 0.9891 & 0.9891 & 0.9892 \\
\hline Precision & 0.9512 & 0.9562 & 0.9511 & 0.9511 & 0.9511 & 0.9513 \\
\hline F-Measure & 0.9577 & 0.9626 & 0.9579 & 0.9579 & 0.9579 & 0.9578 \\
\hline G-Means & 0.9767 & $\mathbf{0 . 9 7 9 6}$ & 0.9769 & 0.9769 & 0.9769 & 0.9767 \\
\hline AUC & 0.9771 & 0.9799 & 0.9774 & 0.9774 & 0.9774 & 0.9773 \\
\hline Train time & $11.05 \mathrm{~s}$ & $4.06 \mathrm{~s}$ & $8.41 \mathrm{~s}$ & $9.03 \mathrm{~s}$ & $10.62 \mathrm{~s}$ & $11.19 \mathrm{~s}$ \\
\hline Test time & $68 \mathrm{~ms}$ & $53 \mathrm{~ms}$ & $73 \mathrm{~ms}$ & $83 \mathrm{~ms}$ & $93 \mathrm{~ms}$ & $109 \mathrm{~ms}$ \\
\hline Features & 42 & 30 & 42 & 42 & 42 & 41 \\
\hline ML & DT & DT & DT & DT & DT & DT \\
\hline
\end{tabular}

algorithms. It is easy to see that GR-BFE has the best $G$-Means, but the Sensitivity is not equal to other algorithms, because other algorithms have a high Sensitivity but their Specificity is very low, resulting in a high false alarm rate, so GR-FBE is still selected.

Table 27. Results of using IG-BFE algorithm on FUZZERS.

\begin{tabular}{|l|c|c|c|c|c|c|}
\hline Metrics & DT & NB & LR & SVM & kNN & ANN \\
\hline Sensitivity & 0.7377 & 0.9835 & 0.9962 & 0.3430 & 0.7988 & 0.8817 \\
\hline Specificity & 0.8729 & 0.8032 & 0.8160 & 0.9516 & 0.8552 & 0.8398 \\
\hline Precision & 0.6534 & 0.6187 & 0.6374 & 0.6971 & 0.6417 & 0.6412 \\
\hline F-Measure & 0.6930 & 0.7596 & 0.7774 & 0.4598 & 0.7117 & 0.7424 \\
\hline G-Means & 0.8025 & 0.8888 & 0.9016 & 0.5714 & 0.8265 & 0.8605 \\
\hline AUC & 0.8287 & 0.8954 & 0.8997 & 0.6473 & 0.8467 & 0.9197 \\
\hline Train time & $4.09 \mathrm{~s}$ & $224 \mathrm{~ms}$ & $20 \mathrm{~s}$ & $157 \mathrm{~s}$ & $30 \mathrm{~ms}$ & $272 \mathrm{~s}$ \\
\hline Test time & $71 \mathrm{~ms}$ & $961 \mathrm{~ms}$ & $153 \mathrm{~ms}$ & $149.8 \mathrm{~s}$ & $456 \mathrm{~s}$ & $197 \mathrm{~ms}$ \\
\hline Features & 35 & 25 & 38 & 12 & 24 & 37 \\
\hline
\end{tabular}

Table 28. Results of using GR-BFE algorithm on FUZZERS.

\begin{tabular}{|l|c|c|c|c|c|c|}
\hline Metrics & DT & NB & LR & SVM & kNN & ANN \\
\hline Sensitivity & 0.7689 & 0.9691 & 0.9960 & 0.7069 & 0.8529 & 0.9148 \\
\hline Specificity & 0.8648 & 0.8079 & 0.8160 & 0.9010 & 0.8513 & 0.8311 \\
\hline Precision & 0.6487 & 0.6210 & 0.6373 & 0.6987 & 0.6507 & 0.6376 \\
\hline F-Measure & 0.7037 & 0.7569 & 0.7773 & 0.7028 & 0.7382 & 0.7514 \\
\hline G-Means & 0.8154 & 0.8849 & 0.9015 & $\mathbf{0 . 7 9 8 1}$ & 0.8521 & 0.8720 \\
\hline AUC & 0.8315 & 0.9026 & 0.9006 & 0.8040 & 0.8932 & 0.9200 \\
\hline Train time & $5.55 \mathrm{~s}$ & $254 \mathrm{~ms}$ & $10.5 \mathrm{~s}$ & $297.3 \mathrm{~s}$ & $19 \mathrm{~ms}$ & $243.7 \mathrm{~s}$ \\
\hline Test time & $80 \mathrm{~ms}$ & $972 \mathrm{~ms}$ & $142 \mathrm{~ms}$ & $306.7 \mathrm{~s}$ & $682.4 \mathrm{~s}$ & $222 \mathrm{~ms}$ \\
\hline Features & 30 & 24 & 38 & 15 & 24 & 34 \\
\hline
\end{tabular}

Similarly, the results of using BFE on EXPLOITS class are shown in the Table 31, Table 32 and Table 33. The best feature reduction algorithm used is CA-BFE, the best machine learning algorithm used is DT, the number of features after being reduced is 37 . Table 34 compares
Table 29. Results of using CA-BFE algorithm on FUZZERS.

\begin{tabular}{|l|c|c|c|c|c|c|}
\hline Metrics & DT & NB & LR & SVM & kNN & ANN \\
\hline Sensitivity & 0.7622 & 0.9817 & 0.9962 & 0.4243 & 0.8489 & 0.8624 \\
\hline Specificity & 0.8684 & 0.8046 & 0.8159 & 0.9539 & 0.8424 & 0.8406 \\
\hline Precision & 0.6528 & 0.6200 & 0.6372 & 0.7493 & 0.6363 & 0.6373 \\
\hline F-Measure & 0.7033 & 0.7600 & 0.7773 & 0.5418 & 0.7274 & 0.7329 \\
\hline G-Means & 0.8136 & 0.8888 & 0.9015 & 0.6362 & 0.8457 & 0.8514 \\
\hline AUC & 0.8421 & 0.9089 & 0.9011 & 0.6891 & 0.8993 & 0.9169 \\
\hline Train time & $6.53 \mathrm{~s}$ & $224 \mathrm{~ms}$ & $9.99 \mathrm{~s}$ & $212.9 \mathrm{~s}$ & $19 \mathrm{~ms}$ & $238.4 \mathrm{~s}$ \\
\hline Test time & $82 \mathrm{~ms}$ & $938 \mathrm{~ms}$ & $165 \mathrm{~ms}$ & $183.8 \mathrm{~s}$ & $606.2 \mathrm{~s}$ & $199 \mathrm{~ms}$ \\
\hline Features & 31 & 20 & 38 & 10 & 22 & 33 \\
\hline
\end{tabular}

Table 30. Compare GR-BFE with other algorithms on FUZZERS attacks.

\begin{tabular}{|l|c|c|c|c|c|c|}
\hline & Origin & BFE & Entropy & Gini & Ridge & Avg \\
\hline Sensitivity & 0.6927 & 0.7069 & 0.9959 & 0.9654 & 0.9959 & 0.9671 \\
\hline Specificity & 0.8737 & 0.9010 & 0.8142 & 0.8154 & 0.8159 & 0.8152 \\
\hline Precision & 0.6404 & 0.6987 & 0.6351 & 0.6294 & 0.6372 & 0.6295 \\
\hline F-Measure & 0.6655 & 0.7028 & 0.7756 & 0.7620 & 0.7777 & 0.7626 \\
\hline G-Means & 0.7780 & $\mathbf{0 . 7 9 8 1}$ & 0.9005 & 0.8872 & 0.9014 & 0.8879 \\
\hline AUC & 0.8060 & 0.8040 & 0.8977 & 0.9093 & 0.8993 & 0.9075 \\
\hline Train time & $8.4 \mathrm{~s}$ & $297 \mathrm{~s}$ & $12.6 \mathrm{~s}$ & $478 \mathrm{~ms}$ & $14.55 \mathrm{~s}$ & $515 \mathrm{~ms}$ \\
\hline Test time & $82 \mathrm{~ms}$ & $307 \mathrm{~s}$ & $161 \mathrm{~ms}$ & $1.69 \mathrm{~s}$ & $161 \mathrm{~ms}$ & $2.81 \mathrm{~s}$ \\
\hline Features & 42 & 15 & 40 & 25 & 33 & 29 \\
\hline ML & DT & SVM & LR & NB & LR & NB \\
\hline
\end{tabular}

Table 31. Results of using IG-BFE algorithm on EXPLOITS.

\begin{tabular}{|l|c|c|c|c|c|c|}
\hline Metrics & DT & NB & LR & SVM & kNN & ANN \\
\hline Sensitivity & 0.9677 & 0.9448 & 0.7694 & 0.9103 & 0.9549 & 0.8750 \\
\hline Specificity & 0.9781 & 0.7718 & 0.9423 & 0.9438 & 0.9826 & 0.9855 \\
\hline Precision & 0.9634 & 0.7117 & 0.8883 & 0.9061 & 0.9703 & 0.9729 \\
\hline F-Measure & 0.9656 & 0.8119 & 0.8246 & 0.9082 & 0.9626 & 0.9214 \\
\hline G-Means & 0.9729 & 0.8539 & 0.8515 & 0.9269 & 0.9687 & 0.9286 \\
\hline AUC & 0.9828 & 0.8917 & 0.9474 & 0.9270 & 0.9833 & 0.9795 \\
\hline Train time & $8.55 \mathrm{~s}$ & $361 \mathrm{~ms}$ & $9.14 \mathrm{~s}$ & $180.8 \mathrm{~s}$ & $16 \mathrm{~ms}$ & $524.8 \mathrm{~s}$ \\
\hline Test time & $78 \mathrm{~ms}$ & $1.98 \mathrm{~s}$ & $228 \mathrm{~ms}$ & $310.9 \mathrm{~s}$ & $778.3 \mathrm{~s}$ & $344 \mathrm{~ms}$ \\
\hline Features & 36 & 25 & 30 & 12 & 29 & 36 \\
\hline
\end{tabular}

the results of using CA-BFE with other algorithms. CAFBE was chosen because CA-BFE has the $G$-Means close to other algorithms (0.9730 compared to 0.9734), but it has the higher Specificity (0.9851 compared to 0.9769). That means the false alarm rate will be lower.

Table 32. Results of using GR-BFE algorithm on EXPLOITS.

\begin{tabular}{|l|c|c|c|c|c|c|}
\hline Metrics & DT & NB & LR & SVM & kNN & ANN \\
\hline Sensitivity & 0.9661 & 0.9521 & 0.8155 & 0.8741 & 0.9562 & 0.8633 \\
\hline Specificity & 0.9784 & 0.7002 & 0.9558 & 0.9502 & 0.9815 & 0.9829 \\
\hline Precision & 0.9639 & 0.6544 & 0.9167 & 0.9128 & 0.9686 & 0.9678 \\
\hline F-Measure & 0.9650 & 0.7757 & 0.8631 & 0.8931 & 0.9623 & 0.9126 \\
\hline G-Means & 0.9722 & 0.8165 & 0.8829 & 0.9114 & 0.9688 & 0.9211 \\
\hline AUC & 0.9810 & 0.8929 & 0.9564 & 0.9122 & 0.9803 & 0.9816 \\
\hline Train time & $5.94 \mathrm{~s}$ & $238 \mathrm{~ms}$ & $4.79 \mathrm{~s}$ & $222.3 \mathrm{~s}$ & $13 \mathrm{~ms}$ & $276.4 \mathrm{~s}$ \\
\hline Test time & $88 \mathrm{~ms}$ & $928 \mathrm{~ms}$ & $126 \mathrm{~ms}$ & $184.6 \mathrm{~s}$ & $716.2 \mathrm{~s}$ & $209 \mathrm{~ms}$ \\
\hline Features & 35 & 24 & 30 & 13 & 29 & 33 \\
\hline
\end{tabular}

The results of using BFE on GENERIC class are shown in the Table 35, Table 36 and Table 37. The best feature reduction algorithm used is IG-BFE, the best machine learning algorithm used is DT, the number of remaining features after being reduced is 34 . Table 38 compares the results of using IG-BFE with other algorithms. 
Table 33. Results of using CA-BFE algorithm on EXPLOITS

\begin{tabular}{|l|c|c|c|c|c|c|}
\hline Metrics & DT & NB & LR & SVM & kNN & ANN \\
\hline Sensitivity & 0.9610 & 0.6752 & 0.8889 & 0.8853 & 0.9562 & 0.8329 \\
\hline Specificity & 0.9851 & 0.9219 & 0.8365 & 0.9443 & 0.9815 & 0.9878 \\
\hline Precision & 0.9746 & 0.8375 & 0.7643 & 0.9045 & 0.9686 & 0.9760 \\
\hline F-Measure & 0.9678 & 0.7476 & 0.8219 & 0.8948 & 0.9623 & 0.8988 \\
\hline G-Means & $\mathbf{0 . 9 7 3 0}$ & 0.7889 & 0.8623 & 0.9143 & 0.9688 & 0.9071 \\
\hline AUC & 0.9822 & 0.8836 & 0.9369 & 0.9148 & 0.9803 & 0.9716 \\
\hline Train time & $6.63 \mathrm{~s}$ & $236 \mathrm{~ms}$ & $3.29 \mathrm{~s}$ & $267.9 \mathrm{~s}$ & $17 \mathrm{~ms}$ & $279.2 \mathrm{~s}$ \\
\hline Test time & $92 \mathrm{~ms}$ & $873 \mathrm{~ms}$ & $122 \mathrm{~ms}$ & $257.1 \mathrm{~s}$ & $687.2 \mathrm{~s}$ & $218 \mathrm{~ms}$ \\
\hline features & 37 & 21 & 24 & 12 & 30 & 35 \\
\hline
\end{tabular}

Table 34. Compare CA-BFE with other algorithms on EXPLOITS.

\begin{tabular}{|l|c|c|c|c|c|c|}
\hline & Origin & BFE & Entropy & Gini & Ridge & Avg \\
\hline Sensitivity & 0.9580 & 0.9610 & 0.9699 & 0.9699 & 0.9593 & 0.9645 \\
\hline Specificity & 0.9795 & 0.9851 & 0.9769 & 0.9769 & 0.9789 & 0.9776 \\
\hline Precision & 0.9653 & 0.9746 & 0.9616 & 0.9616 & 0.9644 & 0.9626 \\
\hline F-Measure & 0.9616 & 0.9678 & 0.9657 & 0.9657 & 0.9618 & 0.9635 \\
\hline G-Means & 0.9687 & $\mathbf{0 . 9 7 3 0}$ & 0.9734 & 0.9734 & 0.9690 & 0.9711 \\
\hline AUC & 0.9687 & 0.9822 & 0.9809 & 0.9809 & 0.9734 & 0.9766 \\
\hline Train time & $11.29 \mathrm{~s}$ & $6.63 \mathrm{~s}$ & $9.94 \mathrm{~s}$ & $8.07 \mathrm{~s}$ & $9.89 \mathrm{~s}$ & $11.78 \mathrm{~s}$ \\
\hline Test time & $116 \mathrm{~ms}$ & $92 \mathrm{~ms}$ & $111 \mathrm{~ms}$ & $111 \mathrm{~ms}$ & $117 \mathrm{~ms}$ & $281 \mathrm{~ms}$ \\
\hline Features & 42 & 37 & 31 & 31 & 37 & 33 \\
\hline ML & DT & DT & DT & DT & DT & DT \\
\hline
\end{tabular}

Thereby, IG-BFE gives the best results.

Table 35. Results of using IG-BFE algorithm on GENERIC.

\begin{tabular}{|l|c|c|c|c|c|c|}
\hline Metrics & DT & NB & LR & SVM & kNN & ANN \\
\hline Sensitivity & 0.9963 & 0.9763 & 0.9878 & 0.9853 & 0.9944 & 0.9901 \\
\hline Specificity & 0.9933 & 0.9966 & 0.9891 & 0.9951 & 0.9934 & 0.9971 \\
\hline Precision & 0.9906 & 0.9952 & 0.9848 & 0.9931 & 0.9908 & 0.9959 \\
\hline F-Measure & 0.9934 & 0.9857 & 0.9863 & 0.9892 & 0.9926 & 0.9930 \\
\hline G-Means & $\mathbf{0 . 9 9 4 8}$ & 0.9864 & 0.9884 & 0.9902 & 0.9939 & 0.9936 \\
\hline AUC & 0.9971 & 0.9922 & 0.9959 & 0.9902 & 0.9962 & 0.9984 \\
\hline Train time & $4.14 \mathrm{~s}$ & $224 \mathrm{~ms}$ & $7.12 \mathrm{~s}$ & $429.7 \mathrm{~s}$ & $17 \mathrm{~ms}$ & $356.1 \mathrm{~s}$ \\
\hline Test time & $66 \mathrm{~ms}$ & $830 \mathrm{~ms}$ & $138 \mathrm{~ms}$ & $175.7 \mathrm{~s}$ & $1246 \mathrm{~s}$ & $283 \mathrm{~ms}$ \\
\hline Features & 34 & 17 & 30 & 36 & 33 & 35 \\
\hline
\end{tabular}

Table 36. Results of using GR-BFE algorithm on GENERIC.

\begin{tabular}{|l|c|c|c|c|c|c|}
\hline Metrics & DT & NB & LR & SVM & kNN & ANN \\
\hline Sensitivity & 0.9965 & 0.9766 & 0.9878 & 0.9831 & 0.9954 & 0.9885 \\
\hline Specificity & 0.9929 & 0.9854 & 0.9914 & 0.9991 & 0.9938 & 0.9981 \\
\hline Precision & 0.9902 & 0.9795 & 0.9880 & 0.9987 & 0.9913 & 0.9973 \\
\hline F-Measure & 0.9933 & 0.9780 & 0.9879 & 0.9908 & 0.9933 & 0.9928 \\
\hline G-Means & 0.9947 & 0.9810 & 0.9896 & 0.9910 & 0.9946 & 0.9932 \\
\hline AUC & 0.9974 & 0.9891 & 0.9960 & 0.9911 & 0.9967 & 0.9977 \\
\hline Train time & $4.57 \mathrm{~s}$ & $303 \mathrm{~ms}$ & $8.06 \mathrm{~s}$ & $445.7 \mathrm{~s}$ & $19 \mathrm{~ms}$ & $357.6 \mathrm{~s}$ \\
\hline Test time & $83 \mathrm{~ms}$ & $1.21 \mathrm{~s}$ & $130 \mathrm{~ms}$ & $259.8 \mathrm{~s}$ & $1136 \mathrm{~s}$ & $290 \mathrm{~ms}$ \\
\hline Features & 36 & 25 & 29 & 37 & 29 & 37 \\
\hline
\end{tabular}

Table 39 represents the effectiveness of the proposed method compared to the classification using full of features in the metrics: G-Means (G-Mea), training time (TrTime) and testing time (TeTime).

Table 40 shows the selected features and machine learning algorithms for each attack type.
Table 37. Results of using CA-BFE algorithm on GENERIC.

\begin{tabular}{|l|c|c|c|c|c|c|}
\hline Metrics & DT & NB & LR & SVM & kNN & ANN \\
\hline Sensitivity & 0.9963 & 0.9815 & 0.9879 & 0.9832 & 0.9950 & 0.9908 \\
\hline Specificity & 0.9923 & 0.9948 & 0.9916 & 0.9991 & 0.9929 & 0.9970 \\
\hline Precision & 0.9892 & 0.9927 & 0.9883 & 0.9987 & 0.9901 & 0.9957 \\
\hline F-Measure & 0.9927 & 0.9871 & 0.9881 & 0.9909 & 0.9926 & 0.9932 \\
\hline G-Means & 0.9942 & 0.9882 & 0.9898 & 0.9911 & 0.9940 & 0.9939 \\
\hline AUC & 0.9973 & 0.9910 & 0.9961 & 0.9911 & 0.9963 & 0.9982 \\
\hline Train time & $5.96 \mathrm{~s}$ & $269 \mathrm{~ms}$ & $5.57 \mathrm{~s}$ & $375 \mathrm{~s}$ & $15 \mathrm{~ms}$ & $347.8 \mathrm{~s}$ \\
\hline Test time & $71 \mathrm{~ms}$ & $1.07 \mathrm{~s}$ & $124 \mathrm{~ms}$ & $198.9 \mathrm{~s}$ & $1140 \mathrm{~s}$ & $265 \mathrm{~ms}$ \\
\hline Features & 37 & 21 & 27 & 34 & 30 & 36 \\
\hline
\end{tabular}

Table 38. Compare IG-BFE with other algorithms on GENERIC.

\begin{tabular}{|l|c|c|c|c|c|c|}
\hline & Origin & BFE & Entropy & Gini & Ridge & Avg \\
\hline Sensitivity & 0.9963 & 0.9963 & 0.9963 & 0.9922 & 0.9963 & 0.9929 \\
\hline Specificity & 0.9894 & 0.9933 & 0.9896 & 0.9942 & 0.9900 & 0.9913 \\
\hline Precision & 0.9854 & 0.9906 & 0.9856 & 0.9919 & 0.9862 & 0.9879 \\
\hline F-Measure & 0.9908 & 0.9934 & 0.9909 & 0.9920 & 0.9912 & 0.9904 \\
\hline G-Means & 0.9929 & $\mathbf{0 . 9 9 4 8}$ & 0.9929 & 0.9932 & 0.9931 & 0.9921 \\
\hline AUC & 0.9967 & 0.9971 & 0.9968 & 0.9984 & 0.9970 & 0.9970 \\
\hline Train time & $8.76 \mathrm{~s}$ & $4.14 \mathrm{~s}$ & $8.2 \mathrm{~s}$ & $395 \mathrm{~s}$ & $10.4 \mathrm{~s}$ & $16 \mathrm{~ms}$ \\
\hline Test time & $82 \mathrm{~ms}$ & $66 \mathrm{~ms}$ & $114 \mathrm{~ms}$ & $305 \mathrm{~ms}$ & $207 \mathrm{~ms}$ & $1945 \mathrm{~s}$ \\
\hline Features & 42 & 34 & 42 & 17 & 41 & 12 \\
\hline ML & DT & DT & DT & ANN & DT & kNN \\
\hline
\end{tabular}

Table 39. The effect of feature reducing with attacks using BFE.

\begin{tabular}{|l|c|c|c|c|c|c|}
\hline Attack & \multicolumn{3}{|c|}{ Full of features } & \multicolumn{3}{c|}{ After using BFE } \\
& G-Mea & TrTime & TeTime & G-Mea & TrTime & TeTime \\
\hline Worms & 0.9068 & $3.84 \mathrm{~s}$ & $48 \mathrm{~ms}$ & 0.9153 & $2.88 \mathrm{~s}$ & $31 \mathrm{~ms}$ \\
\hline Shellcode & 0.9053 & $3.97 \mathrm{~s}$ & $48 \mathrm{~ms}$ & 0.9595 & $2.58 \mathrm{~s}$ & $43 \mathrm{~ms}$ \\
\hline Backdoor & 0.9652 & $8.73 \mathrm{~s}$ & $78 \mathrm{~ms}$ & 0.9799 & $3.07 \mathrm{~s}$ & $53 \mathrm{~ms}$ \\
\hline Analysis & 0.9138 & $6.31 \mathrm{~s}$ & $102 \mathrm{~ms}$ & 0.9422 & $0 \mathrm{~ms}$ & $408 \mathrm{~s}$ \\
\hline Reconnai & 0.9545 & $7.65 \mathrm{~s}$ & $61 \mathrm{~ms}$ & 0.9802 & $4.26 \mathrm{~s}$ & $41 \mathrm{~ms}$ \\
\hline DoS & 0.9767 & $11.1 \mathrm{~s}$ & $68 \mathrm{~ms}$ & 0.9796 & $4.06 \mathrm{~s}$ & $53 \mathrm{~ms}$ \\
\hline Fuzzers & 0.7780 & $8.44 \mathrm{~s}$ & $82 \mathrm{~ms}$ & 0.7981 & $297 \mathrm{~s}$ & $307 \mathrm{~s}$ \\
\hline Exploits & 0.9687 & $11.3 \mathrm{~s}$ & $116 \mathrm{~ms}$ & 0.9730 & $6.63 \mathrm{~s}$ & $92 \mathrm{~ms}$ \\
\hline Generic & 0.9929 & $8.76 \mathrm{~s}$ & $82 \mathrm{~ms}$ & 0.9948 & $4.14 \mathrm{~s}$ & $66 \mathrm{~ms}$ \\
\hline
\end{tabular}

Table 40. Results of feature reducing with each attack type using BFE algorithm.

\begin{tabular}{|l|l|c|}
\hline Attack & ID of features selected & ML \\
\hline Worms & $2,3,4,5,6,7,8,9,10,11,12,13,15,16,17,18,19,20,21,22$, & DT \\
& $23,24,25,26,27,28,30,31,33,34,36,37,38,39,40,41,42$, & \\
& 43 & \\
\hline Shellcode & $4,5,6,7,8,9,11,12,13,14,15,16,17,18,19,21,24,27,28$, & DT \\
& $29,30,31,32,33,35,36,37,38,39,40,42,43$ & \\
\hline Backdoor & $2,3,4,6,7,8,9,10,11,12,13,14,15,16,17,18,19,21,23,24$, & DT \\
& $25,26,27,28,29,31,33,34,37,38,39,40,42,43$ & \\
\hline Analysis & $2,3,5,6,7,9,11,12,14,15,16,17,18,19,20,21,24,31,33$, & kNN \\
& $38,39,40,43$ & \\
\hline Reconna- & $2,3,4,5,6,7,8,10,11,12,13,16,18,19,20,21,22,23,24,25$, & DT \\
issance & $26,27,28,29,31,33,34,35,36,37,38,39,40,41,43$ & \\
\hline DoS & $2,3,4,8,9,12,14,15,16,17,19,20,21,23,24,25,26,28,29$, & DT \\
& $30,32,33,34,35,36,39,40,41,42,43$ & \\
\hline Fuzzers & $2,4,5,7,9,10,11,13,14,19,24,28,37,38,39$ & SVM \\
\hline Exploits & $2,3,4,5,6,7,9,10,11,13,14,15,16,17,19,20,21,22,23,24$, & DT \\
& $25,26,27,28,29,30,32,33,34,35,37,38,39,40,41,42,43$ & \\
\hline Generic & $2,3,4,7,8,9,10,11,12,13,14,15,16,18,20,21,22,24,25$, & DT \\
& $28,29,30,32,33,34,35,36,37,38,39,40,41,42,43$ & \\
\hline
\end{tabular}

\section{CONCLUSION}

From the experimental results, some conclusions are drawn as follows:

(1) Class distribution in the network intrusion detection system is very imbalanced, so using 
Accuracy metric to evaluate the classification quality of a model is incorrect. Therefore, the use of metrics such as: $F-$ Measure and $G-$ Means proved it.

(2) The evaluation results on the UNSW-NB15 dataset show that this dataset has many complex patterns, especially at the attack classes such as WORMS, ANALYSIS and FUZZERS.

(3) Dimensional reduction of data not only reduces computational cost but also improves classification quality in Intrusion Detection Systems.

(4) The use of the IG-BFE, GR-BFE and CA-BFE algorithms to reduce data dimensions is better than the other known algorithms.

(5) For each different type of attack, different features and machine learning algorithm will be chosen so that the performance of the intrusion detection system is improved at the best.

At the same time, the experimental results also set out issues that need to be further studied, especially the contents:

(1) Research using ensemble methods such as boosting, bagging, voting, stacking, and etc, can help improve classification quality.

(2) The UNSW-NB15 dataset is still quite new, it has not been used by many scholars in their studies. Therefore, there are limitations when comparing results with other studies.

\section{References}

[1] SH Kok, Azween Abdullah, NZJhanjhi, and Mahadevan Supramaniam. A review of intrusion detection system using machine learning approach. International Journal of Engineering Research and Technology, ISBN 0974-3154, 12(1):8-15, 2019.

[2] Al-Jarrah O. Y., Siddiqui A., et al. Machine-learningbased feature selection techniques for large-scale network intrusion detection. In Distributed Computing Systems Workshops, 2014 IEEE 34th International Conference on, pages 177-181, 2014.

[3] B.M. Aslahi-Shahri, R. Rahmani, M. Chizari, A. Maralani, M. Eslami, M.J. Golkar, and A. Ebrahimi. A hybrid method consisting of ga and svm for intrusion detection system. Neural Comput. Appl., 27:1669-1676, 2016.

[4] W. Xingzhu. Aco and svm selection feature weighting of network intrusion detection method. Analysis, 9:129-$270,2015$.
[5] M.S. Rani and S.B. Xavier. A hybrid intrusion detection system based on c5.0 decision tree and one-class svm. Int. J. Curr. Eng. Technol., 5:2001--2007, 2015.

[6] W.A.H.M. Ghanem and A. Jantan. Novel multi-objective artificial bee colony optimization for wrapper based feature selection in intrusion detection. Int. J. Adv. Soft Comput. Appl., 8:70-81, 2016.

[7] N. Acharya and S. Singh. An iwd-based feature selection method for intrusion detection system. Soft Comput., 22:1--10, 2017.

[8] S. Ganapathy, P. Vijayakumar, P. Yogesh, and A. Kannan. An intelligent crf based feature selection for effective intrusion detection. Int. Arab J. Inf. Technol., 13:44--50, 2016.

[9] M.A. Ambusaidi, X. He, P. Nanda, and Z. Tan. Building an intrusion detection system using a filter-based feature selection algorithm. IEEE Trans. Comput., 65:2986-2998, 2016.

[10] A.I. Madbouly and T.M. Barakat. Enhanced relevant feature selection model for intrusion detection systems. Int. J. Intell. Eng. Inform., 4:21-45, 2016.

[11] J. W. Han, M. Kamber, and J. Pei. Data Mining Concepts and Techniques. 3rd Edition, Morgan Kaufmann Publishers, Waltham, 2012.

[12] R. P. Espíndola and N. F. F. Ebecken. On extending fmeasure and g-mean metrics to multi-class problems. In WIT Transactions on Information and Communication Technologies, volume 35. WIT Press, 2005.

[13] Yuk Ying Chung and Noorhaniza Wahid. A hybrid network intrusion detection system using simplified swarm optimization (sso). In Applied Soft Computing 12, pages 3014-3022. Elsevier, 2012.

[14] Hoang Ngoc Thanh, Tran Van Lang, and Hoang Tung. A machine learning approach to classify types of attacks in network intrusion detection system. In Proceedings of the 9th National Conference on Fundamental and Applied IT Research (FAIR'9), pages 502-508, Vietnam, 2016.

[15] Hoang Ngoc Thanh and Tran Van Lang. Feature selection based on information gain to improve performance of network intrusion detection systems. In Proceedings of the 10th National Conference on Fundamental and Applied IT Research (FAIR'10), pages 823-831, Vietnam, 2017.

[16] Hoang Ngoc Thanh and Tran Van Lang. Creating rules for firewall use of decision tree based ensemble techniques. In Proceedings of the 11th National Conference on Fundamental and Applied IT Research (FAIR'11), pages 489-496, Vietnam, 2018.

[17] N. Moustafa and J. Slay. Unsw-nb15: A comprehensive dataset for network intrusion detection. In Paper presented at the Military Communications and Information Systems Conference, 2015. 